Classification

Physics Abstracts

47.25Q

\title{
Rayleigh-Bénard convective structures in a cylindrical container
}

\author{
V. Croquette, M. Mory and F. Schosseler \\ SPSRM, Orme des Merisiers, 91191 Gif-sur-Yvette Cedex, France
}

(Reçu le ler août 1982, accepté le 19 novembre 1982)

\begin{abstract}
Résumé. - Dans cet article nous faisons une étude des différentes structures convectives que l'on peut observer dans une géométrie cylindrique telle que le diamètre soit égal à 20 fois la hauteur. Pour un nombre de Prandtl de 380 , ces structures apparaissent désordonnées mais stationnaires (après une phase de relaxation). Nous montrons que ces structures désordonnées sont plus stables que les solutions axisymétriques et nous indiquons comment les conditions aux limites jouent un rôle essentiel sur la stabilité de ces dernières. Enfin, nous considérons deux types de défauts, que l'on rencontre fréquemment dans les structures désordonnées. Nous les étudions par l'intermédiaire de leur champ de vitesse obtenu par vélocimétrie laser. Nous montrons que cette méthode nous permet une comparaison quantitative avec des théories récentes.
\end{abstract}

\begin{abstract}
In this article we investigate the various convective patterns that appear in a cylindrical container with a diameter 20 times larger than its depth. It turns out that, with a fluid having a Prandtl number of 380 , these patterns are disordered and stationary (after a relaxation process). We show that these disordered structures are favoured with respect to regular ones like axisymmetric roll patterns and we demonstrate that the boundary conditions play an essential part in this instability. At last we consider two kinds of defects commonly encountered in these disordered structures and we study them through their velocity field obtained by laser Doppler anemometry. We show that this method enables quantitative comparison with recent theories to be done.
\end{abstract}

1. Introduction. - The Rayleigh-Bénard instability is one of the most convenient ways to experimentally test the new ideas which have been proposed concerning the onset of turbulence in fluid flows [1]. Certainly, the experiments which are the best understood at present are those where the number of convective rolls is small; this situation arises when the horizontal dimensions of the container are of the same order of magnitude as its depth. In such containers the spatial degrees of freedom of the convective structures are frozen out so that the turbulence sets in after a few temporal instabilities have appeared [2]. If we consider the opposite situation, that is when the horizontal dimensions are significantly larger than the depth, the experimental results are far from being well understood. Even in the simple unimodal roll convective state, that is when the structure which sets in after the conducting state may be described by a single system of rolls, periodic in one direction only, the convection appears to be disordered and in some cases is non-stationary, even when the Rayleigh number $R$ is very close to its critical value $R_{\mathrm{c}}$ [3]. The occurrence of turbulence is not so well defined either; one possible behaviour in experiments is a continuous grow-up of noise versus $R$ and an exponential decay of its spectrum versus frequency [4]. Nevertheless some experiments [5] do not at all follow these rules.

We think that this puzzling situation is even complicated by the fact that such an elementary information as the convective pattern is often not even known. Nevertheless the turbulence is believed to be associated with spatial instabilities like defect motions [6]. If we consider the simple analogy between convective structures and solid-state crystals, this turbulence is, in a way, equivalent to the melting of the crystal. However convective structures are more involved since they are not governed by any variational principle as are crystals. In this situation the disordered structures, also called « textures", might be non-stationary, leading to a turbulent state. 
In this rather puzzling situation, the high Prandtl number $P$ case appears to be the simplest case : theoretically the limit $P \rightarrow \infty$ allows the inertial effect to be ignored, which results in a very convenient simplification of the Boussinesq equations [7]. Experimentally we demonstrate that if the Rayleigh number does not exceed a few times its critical value, natural structures tend to relax towards a metastable state which is stationary. This allows us to investigate the stability of these structures, the topology of the defects encountered and also finer details of these defects. The present study deals with structures encountered in a cylindrical container. First we investigate the stability of axisymmetric and non-axisymmetric structures depending on the lateral boundary conditions, then we present a careful study of two kinds of defects when they are stationary. The first kind is a dislocation, the second is a grain boundary. We emphasize that this second kind of defect is really common in texture-like structures since it allows two sets of rolls to be joined. It turns out that the preferred angle between the two sets of rolls is $90^{\circ}$.

2. Experimental set-up. - All the experiments were carried out in a cylindrical container $4 \mathrm{~mm}$ high and $80 \mathrm{~mm}$ in diameter. The fluid is silicon oil with a viscosity of $v=43 \mathrm{cst}$ (at $32^{\circ} \mathrm{C}$ ), a thermal diffusivity $D_{\text {th }}=1.13 \times 10^{-3} \mathrm{~cm}^{2} / \mathrm{s}$ and thus with a high Prandtl number : $P=380$. In order to achieve good thermal boundary conditions, we use a massive copper plate, at the bottom of the container and a sapphire monocrystal at the top. We regulate the temperature of these plates by circulating water, the temperature difference is monitored with an accuracy of $0.02{ }^{\circ} \mathrm{C}$. (The critical temperature difference is $13.1^{\circ} \mathrm{C}$.) The container itself is a plexiglass ring, this material has nearly the same thermal conductivity as the oil.

The convective structures may be studied by two optical methods : shadowgraphic images and laser Doppler anemometry. This is possible since the copper

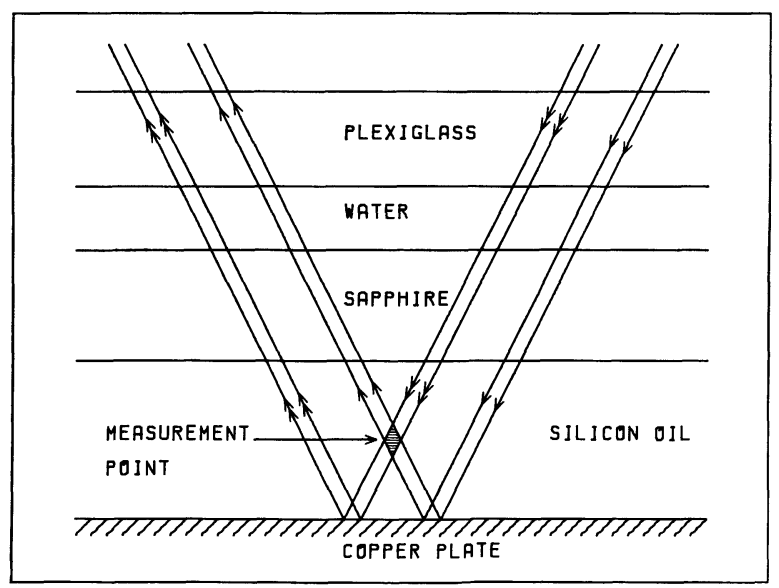

Fig. 1a. - Sketch of the laser Doppler anemometry imaging. This method enables us to determine the vertical velocity component.

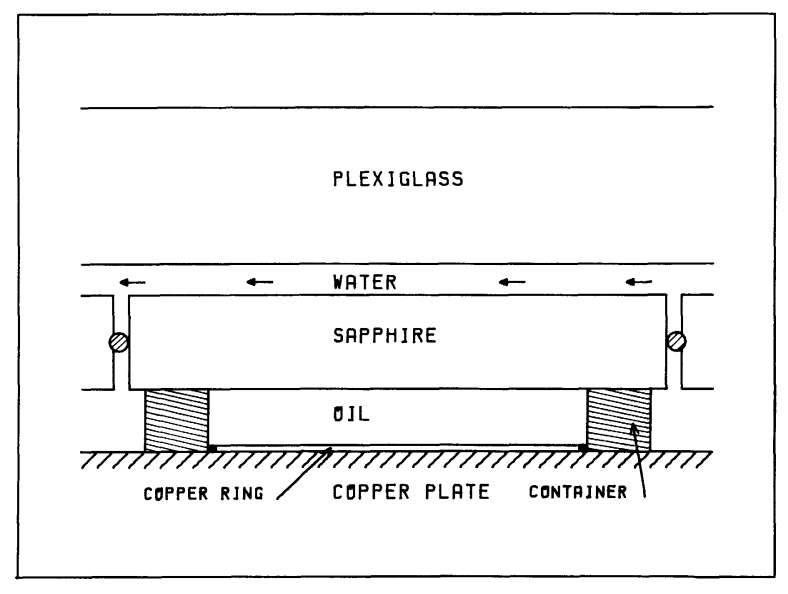

Fig. 1b. - Sketch of the triggering device to obtain axisymmetric structures. The copper ring in good thermal contact with the copper plate produces a horizontal gradient inducing the axisymmetric rolls. The vertical scale is about 2.5 times larger than the horizontal one.

plate is polished nearly to a mirror finish; shadowgraphic images are obtained with a parallel vertical light beam reflected of the mirror and projected onto a screen. Laser Doppler anemometry is achieved by two parallel laser beams entering the cell with an angle of approximately $45^{\circ}$. The point of measurement is defined as the crossing of the direct upper beam and the reflected lower beam (see Fig. 1a), so that we measure the vertical velocity component in the midheight of the container (with our optical configuration). Two Bragg modulators are inserted in the light paths so that the velocity as well as its sign can be determined. With these two methods, we can determine simultaneously the overall roll pattern (with the focalization images) and the velocity at one point. By using a semi-automatic scanning of the point of measurement, we are able to determine the velocity field of a roll pattern as explained by J. P. Gollub in [17].

A last facility of our optical arrangement is the possibility of inducing particular structures. By using the method described by Chen and Whitehead [8], we may prepare a structure corresponding to a prescribed grid. A description of the process may be found in [9].

3. Natural structures in a cylindrical container. We call " natural structures » structures which are not obtained by any induction process but appear when we increase the temperature gradient very slowly $\left(10^{-3} \Delta T_{\mathrm{c}}\right.$ per min.) above its critical value. These structures are rather puzzling, as can be seen on figure 2, where they are clearly disordered; even more surprising is the fact that the structure changes with each experiment. Considering the various patterns we have obtained, we have noticed the following common properties. The rolls always end perpendicular to the sidewalls, the width of the rolls is fairly constant over all the structure [10] and finally the structures may usually be described as a set of fairly regular roll patches linked by several kinds of defects. 


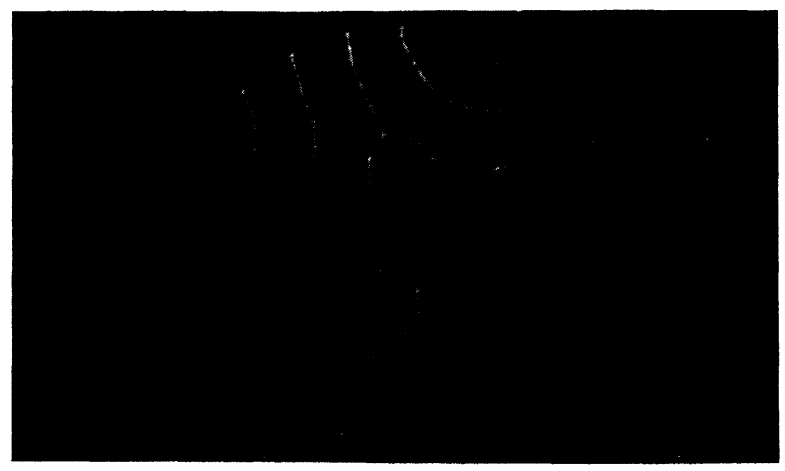

Fig. 2. - Shadowgraphic image of a texture like structure. This pattern is stationary at $\varepsilon=1$. (White lines correspond to descending streams, black lines to ascending streams.)

We have observed that these structures exhibit a transient time dependence : after the convection has set up during the slow rise of the temperature gradient, the temperature difference is kept constant; nevertheless the pattern undergoes a very slow evolution. More precisely this evolution consists in rapid events (time scale $30 \mathrm{~min}$.) separated by long periods during which no further modification seems to occur. The phenomenon lasts for a few days, the periods of rest increasing with time until no more events occur (at least over a time of three weeks). This suggests that the pattern has reached some kind of equilibrium state. It is rather difficult to say if this equilibrium is stable or unstable; we think it is metastable since a great number of equilibrium states are possible for a definite temperature difference.

The rapid events consist in motion of defects, destruction or apparition of a roll. The long periods of rest are probably associated with the diffusion of the stresses in the structure. It is interesting to remark that M. M. Chen and J. A. Whitehead [8] have already observed these disordered structures : in their paper [8], which concerns the induction of a parallel roll structure in a cylindrical container and the study of its stability, pictures of pattern evolution are given. When the induced structure has a wavevector different from the preferred value, the pattern undergoes an evolution involving structural instabilities (like the zig-zag type) leading to a texture-like structure very similar to that presented in figure 2 .

4. Axisymmetry considerations. - We may wonder why the structures we observed show such a disorder and are not similar to the axisymmetric structures observed by E. L. Koschmieder [11] and by Hoard, Robertson and Acrivos [12]. Actually, we cannot fully answer this question but we will demonstrate that. with our experimental conditions, axisymmetric patterns are unstable : If we induce a perfectly axisymmetric roll structure in our container, we indeed observe that it stay during the few minutes following the induction process, but rapidly (within half an hour) the roll adjacent to the sidewall breaks uniformly and

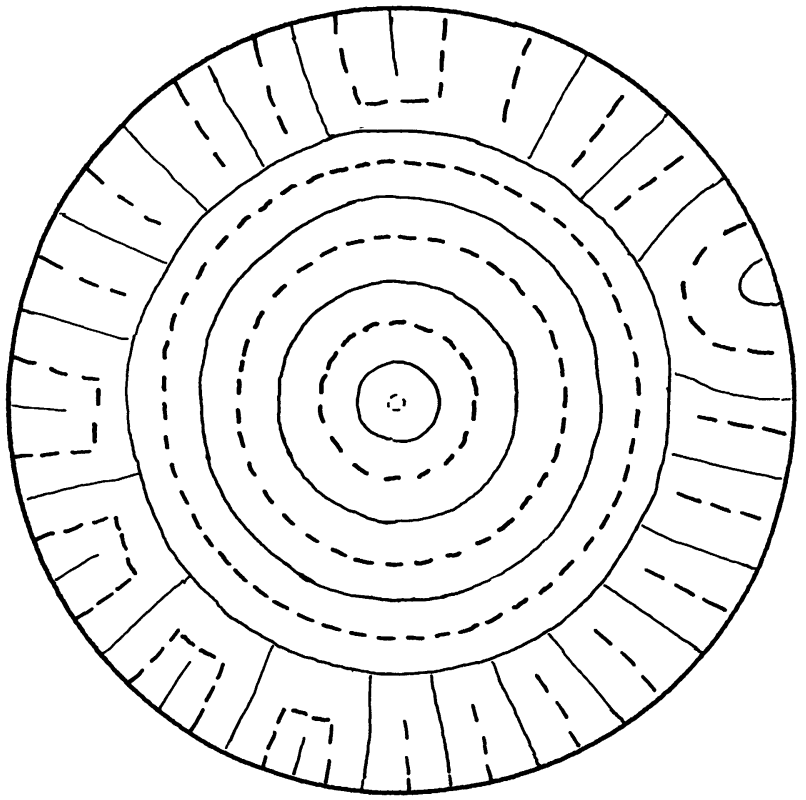

Fig. 3. - Instability of an axisymmetric pattern : when we induce an axisymmetric structure, the roll near the sidewall rapidly breaks down. This structure was observed half an hour after the induction at $\varepsilon=1.5$.

gives rise to a set of small rolls perpendicular to the sidewall. (This clearly appears in Fig. 3.) This process keeps going on, but this time the evolution is slow;

After several days the annulus pattern is completely destroyed leading to a structure similar to a natural one. Such an instability of axisymmetric structures has already been observed by Stork and Muller [13] and more recently by K. R. Kirkchatz, et al. [14]. This result is also in agreement with the prediction of M. C. Cross [15]. In fact the pattern is governed by the boundary conditions at the sidewall : we observe that the rolls end perpendicularly to the sidewall and we notice that this was not the case in Koschmieder's experiment [11]. We tried to reproduce the axisymmetric pattern by altering our boundary conditions : by gluing a copper ring all along the sidewall (see the

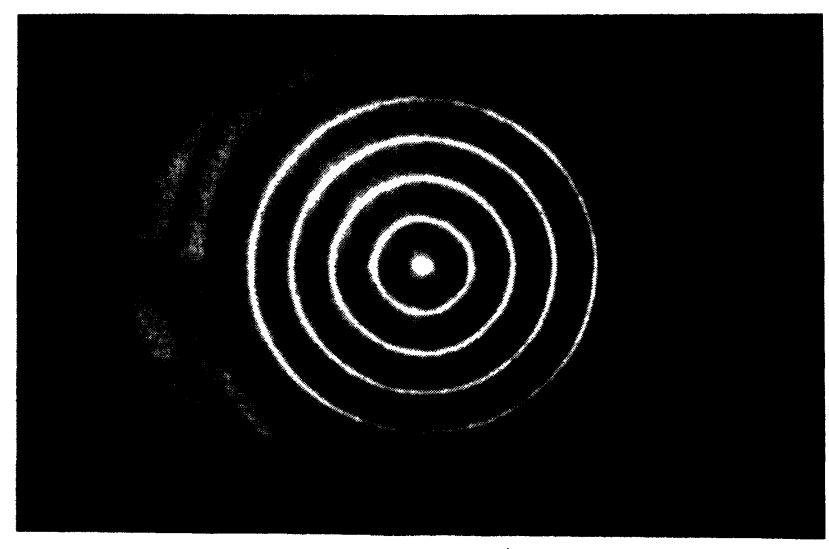

Fig. 4. - Axisymmetric pattern stabilized by the copper $\operatorname{ring}(\varepsilon=0.2)$. 
sketch of Fig. 1b), which is, in thermal contact with the copper plate, one produces a small horizontal thermal gradient at the sidewall. As expected, with this modification we obtain an axisymmetric pattern (see the Fig. 4) and we observe subcritical rolls near the sidewall exactly like those of reference [11]. These subcritical rolls clearly appear on the velocity recordings $a$ ) and $b$ ) of figure 5. This experiment demonstrates the influence of the sidewall in defining the structure. In our case (without the copper ring) where the conductivity of the sidewall is nearly the same as that of the fluid, we found that the condition that the rolls end perpendicularly to the sidewall is fundamental. This situation has already been observed in different geometries $[16,17]$ our observation confirms the tendency of the rolls to align perpendicular to the boundary even in an axisymmetric cell. We may ask whether, in the case of perfectly conducting sidewall, the situation would still be the same ? It is difficult to reproduce this situation in a real experiment; however we may consider that the experiments of

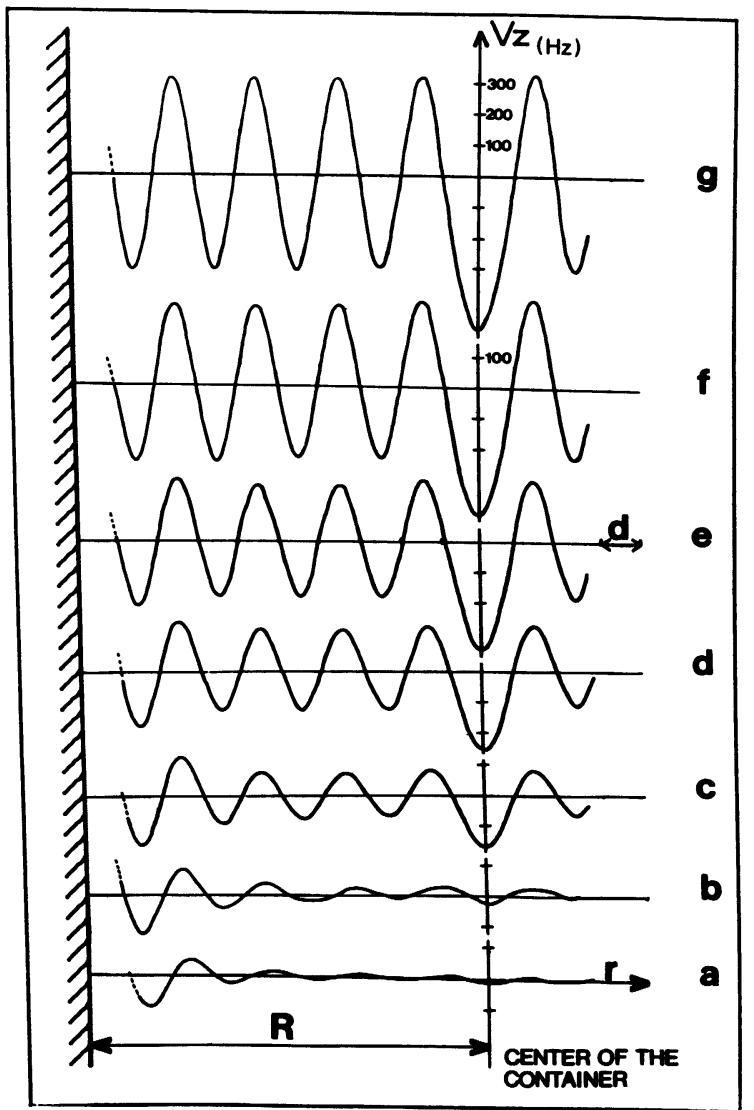

Fig. 5. - Vertical velocity component versus the distance to the centre in an axisymmetric structure. $a$ ) and $b$ ) show subcritical rolls. Notice the important descending flow in the centre of the pattern $(c), d), e), f), g)$ ). a) corresponds to $\varepsilon=-0.069, b)$ to $\varepsilon=-0.036, c)$ to $\varepsilon=0.004, d)$ to $\varepsilon=0.023, d)$ to $\varepsilon=0.040, f$ ) to $\varepsilon=0.077, g$ ) to $\varepsilon=0.111$ $(100 \mathrm{~Hz} \leftrightarrow 30 \mu \mathrm{m} / \mathrm{s})$.
K. R. Kirkchartz et al. in a cylindrical container [14] correspond to a case which is closer to perfectly conducting sidewalls than ours since their glass sidewall is likely to have a conductivity about 8 times higher than that of their fluid K. R. Kirkwharts et al. also found that the axisymmetric structure was unstable when they applied a steady temperature gradient but they observed that the structure is in fact made up of well-aligned rolls. In their case the rolls do not seem to bend in order to end perpendicularly to the sidewalls. This boundary condition is less important but it is difficult to say if this is related to the conductivity of the sidewalls or rather to their small aspectratio $(\Gamma=6)$.

5. Amplitude function in the axisymmetric pattern. We have just seen how it is possible to stabilize an axisymmetric pattern. This allows the annulus at the centre of the cell, which constitutes a singularity in the flow to be studied using the laser Doppler anemometry. The experiment is greatly facilitated by the symmetry of the structure : we have measured the vertical velocity component along a radius of the container. Figure 6 presents these velocity recordings for different temperature gradients.

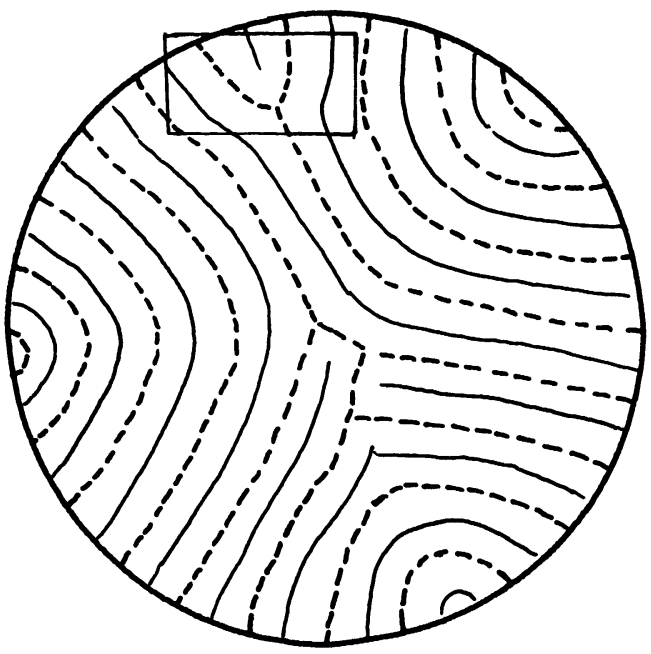

Fig. 6. - Overal pattern of the structure in which we have studied a dislocation. The velocity field corresponds to the rectangle. Dotted lines stand for cold stream and full lines for hot streams.

The velocity presents a maximum at the centre of the cell, and this result is in contradiction with the expectation of G. Ahlers et al. [18] who indicate that the amplitude of the convection $A$ should vanish at the centre of the cell $A(r=0)=0$ when the radius $L$ of the container is infinite, and should present a minimum in the case of a finite radius $A(r=0)=0(1 / \operatorname{Ln} L)$ 
( ${ }^{1}$. In our experiment : $L=10 d$, so according to reference [18] we should expect at least a visible minimum which is clearly absent. We think that this idea of an amplitude minimum at the centre of the container came from the choice of a peculiar solution made by S. N. Brown and K. Stewartson [19]. In a complementary paper [20] they studied a second kind of solution which should present a "weak singularity at the origin ". Unfortunately the authors focused their work more especially on the modification of the convection threshold (which is barely accessible in experiments) rather than on the shape of this solution and its evolution with the reduced Rayleigh number.

We have also checked that the maximum we observe is not a spurious effect due to the triggering copper ring : the maximum is also present when we induce an axisymmetric structure in the container without the triggering device.

We may also notice that on figure $6 c$ the maximum associated with the ombilic has a different nature than the maximum associated with the triggering system : the triggering system induces an amplitude maximum which recovers its normal value with an exponential variation taking place over a length equal to the influence length $\xi$ [21]. On the other hand the maximum associated with the ombilic does not seem to alter very much the amplitude of the adjacent roll or perhaps on a smaller length scale.

6. Defects study. - We have seen that the natural structures that we observe contain defects, as E. D. Siggia and A. Zippelius have pointed out [22]. The dynamics of these defects appears to be essential for the pattern evolution that we observe. However the defects are never isolated and have various aspects. In this article we will focus on a description of the experimental situation and on the static properties of these defects.

We have noticed three main kinds of defects : dislocations, disclinaisons and grain boundaries between two perpendicular roll structures, this last kind being the most frequent in our cylindrical container.

The dislocations and disclinaisons are usually located close to the sidewalls and if this is not the case they move relatively rapidly towards the sidewalls where they either collapse or are trapped. They move

( $\left.{ }^{1}\right)$ In their article G. Ahlers et al. did not completely rule out the possibility of a maximum at the centre of the container. In their appendix B they proposed (p. 327) a possible function for the amplitude in the case of the singularity described in [20]. However this function is too simple to be able to agree with our results (Fig. 5); especially the amplitude at the centre should behave like $\varepsilon^{1 / 2}$ as it does at all other points in the container. On the contrary we find that the amplitude at the centre of the container behaves to a first approximation like a power law with an exponent smaller than $1 / 2$ since the ratio $A(r=0) / A(r \neq 0)$ diverges when $\varepsilon \rightarrow 0$. usually mainly by gliding. That is they move perpendicularly to the roll axis, and, this may be understood if we refer to the ideas developed by Y. Pomeau et al. in [23] : they show that the climbing velocity (a climbing motion occurs when the defect moves along the roll axis) introduced in [22] vanishes when the wavevector of the structure has the optimal value for the temperature gradient. In our experiment the rolls ending perpendicularly to the sidewalls enable a continuous adjustement of the wavenumber to be obtained so that $k=k_{\mathrm{op}}$, that is $\delta q=0$ in the notation of E. D. Siggia; thus the climbing motion are not favoured. On the other hand the rolls are often bent (see Fig. 2) and in this case Y. Pomeau et al. have demonstrated the possibility of gliding. Yet the motions that we observe are highly modulated by the roll structure. Their velocity is very small (one $\Lambda$ in a few hours) and far from being uniform. These gliding motions ceased when the Rayleigh number is high. In the following paragraph we shall discuss the velocity field around a dislocation : we conjecture that the gliding motion is related to the topology of this velocity field.

7. Velocity field around a static dislocation. - Our velocity measurement method is not sufficiently fast to provide a quasi-instantaneous map of the dislocation during its motion. Thus we report the velocity map of a static dislocation; as we have said in such a situation the dislocation is trapped near the sidewall. Figure 6 shows the overall structure in the cylindrical container; the encircled area is the dislocation region where we have performed the velocity measurements. The velocity map is reproduced in figure 7 , in fact it covers only the core of the dislocation. We see that the amplitude of the convection is only altered in three areas : in region $\mathrm{B}$, the velocity reaches a maximum greater than anywhere in the structure; on the other hand, in region $\mathrm{A}$ and $\mathrm{A}^{\prime}$ we find two saddle points, that is there are two minima in the line of maxima associated to the descending flow. At first glance this velocity field is surprising : Considering the defect analysis $[22,23]$ a dislocation may be seen as a phase discontinuity along a segment of line (associated to the extra roll pair). In order to avoid a singularity in the physical fields the intensity of convection ought to vanish at the core of the dislocation. We expected that the velocity field would reflect this idea by showing a reduced velocity at the core of the dislocation. This minimum should extend on the scale of a coherence length $\xi_{\|}(0.6 d$ at $\varepsilon=0.41)$ in the direction perpendicular to the roll axis and on a length $\xi_{\perp}=\left(\xi_{\|} / 2 k c\right)^{1 / 2}$ in the direction of the roll axis $\left(\xi_{\perp}=0.31, \varepsilon=0.41\right)$ $[19,22]$. As this minimum extend further in the direction perpendicular to the roll, the occurrence of $A$ and $A^{\prime}$ is quite natural. Nevertheless it is more surprising that we observe a maximum of velocity near a place we identify as the core of the dislocation. P. Manneville suggests that in fact this maximum 


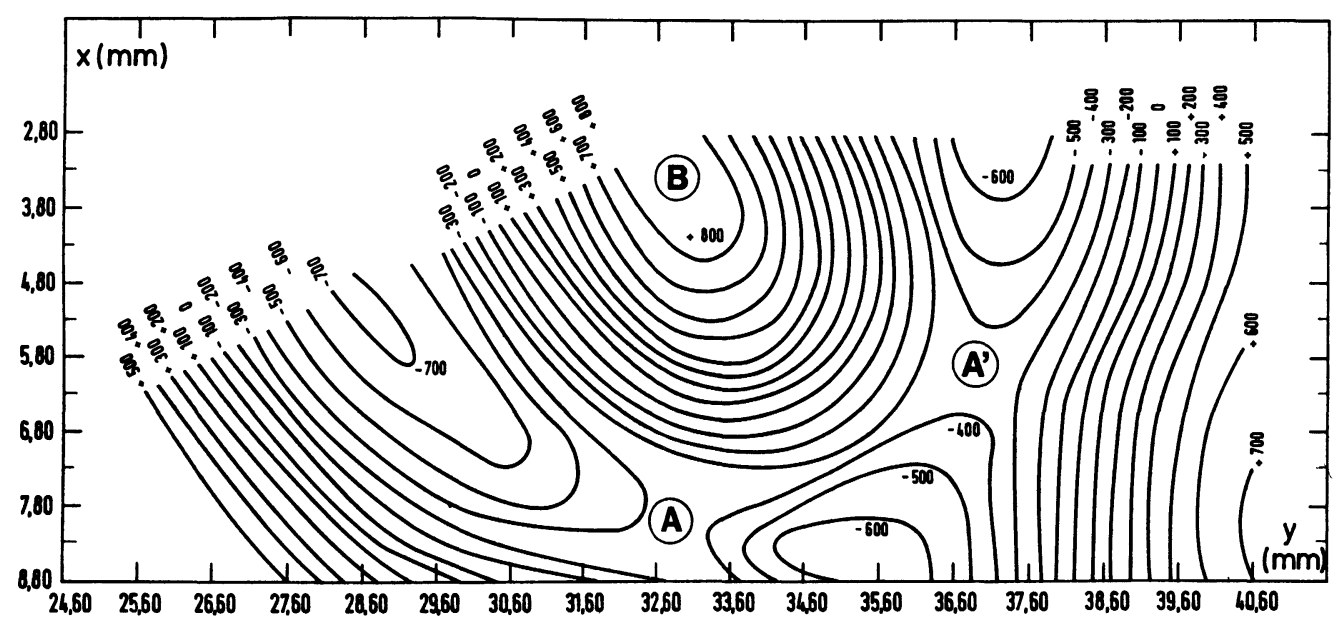

Fig. 7. - Velocity field of the core of a dislocation. The lines are isovelocity curves. This field is from 500 velocity measurements. $(100 \mathrm{~Hz} \leftrightarrow 30 \mu \mathrm{m} / \mathrm{s})$.

arises from the rapid variation of the amplitude : in the direction of the roll axis the amplitude must go from zero to its normal value; this amplitude variation is governed by a fourth-order differential equation. Thus the amplitude recovers its normal value on a length scale $\xi_{\perp}$ and after an overshoot. In fact the maximum $B$ is probably the physical sign of this overshoot which also appears in P. Manneville's simulations (amplitude equations) and in the temperature fields of reference [22] (3d-Boussinesq equations). In this last case another maximum occurs in the roll which separates in two; we have not seen it but it might be attenuated by the sidewall in our experiment. However if this is the case it would be interesting to compare the amplitude overshoots for example with theoretical calculations; this would give some insight on the amplitude behaviour in the direction of the roll axis which has not been studied experimentally so far.

In the gliding motion, we have noticed that the two saddle points $\mathrm{A}$ and $\mathrm{A}^{\prime}$ play an important roll. They constitute weak points in the roll structure so that they enable the rolls to break during the gliding motion. We think that velocity measurements around the saddle points would be interesting in order to describe more completely the gliding motion.

We also plan to measure the velocity map far from the core of the dislocation in order to compare it with the deformation field calculated in [22]; such a measurement has already been made for a similar instability of a nematic under an elliptical shear [25].

8. Velocity map at the boundary between two perpendicular systems of rolls. - We have noticed that this kind of defect occurred very often in rectangular containers. This defect enables the rolls to end perpendicular to the four sidewalls. In the cylindrical containers we have noticed that the pattern relaxes to a situation where these defects are dominant.

The structure of this defect is quite simple : let us consider the roll structure of figure $8:$ at the left of a vertical line we find a roll structure which we call $A$

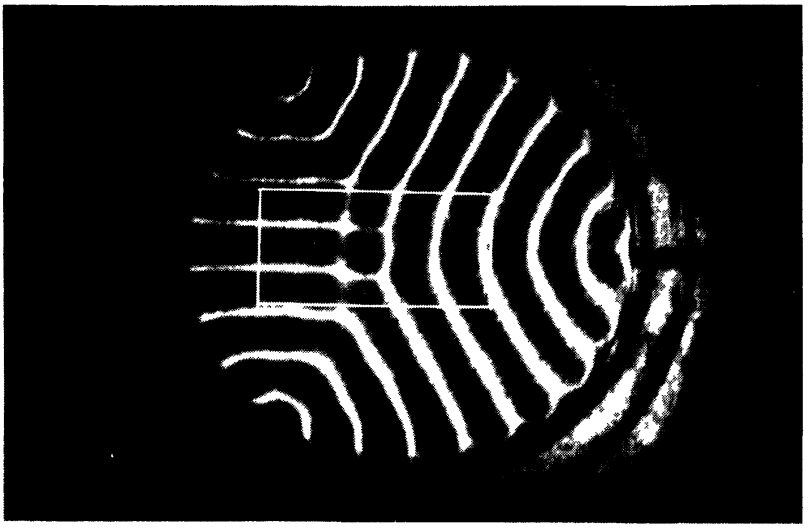

Fig. 8. - Grain boundary in a cylindrical container. This structure corresponds to the evolution of disclinaison structure originally induced with a grid having a three fold symmetry. This structure is unstable leading to this grain boundary easy to study since it is located in the middle of the container $(\varepsilon=0.22)$.

and at the right of this line another roll structure B perpendicular to $\mathrm{A}$. We will see that this line is not so clearly defined, the B roll structure penetrating the horizontal roll and vice versa.

This time the defect is stable in the middle of the container, and we can see the overall structure on figure 8 ; the rectangle defined by the white lines bounds the area where we have performed the velocity map given on figure 10. This structure was obtained at $\varepsilon=0.22$ and was stationary (accurate velocity measurements demonstrate that this structure remains unchanged, even to the smallest details, during at least three weeks). In this velocity map we clearly distinguish the B roll structure on the right but the A roll structure is less obvious on the velocity map than on the focalization pictures of figure 8 . We have tried to evidence this phenomenon by analysing the centre of the velocity map of figure 9 , we have determined the amplitude of both sets of rolls in a strip limited to $y=32 \mathrm{~mm}$ 


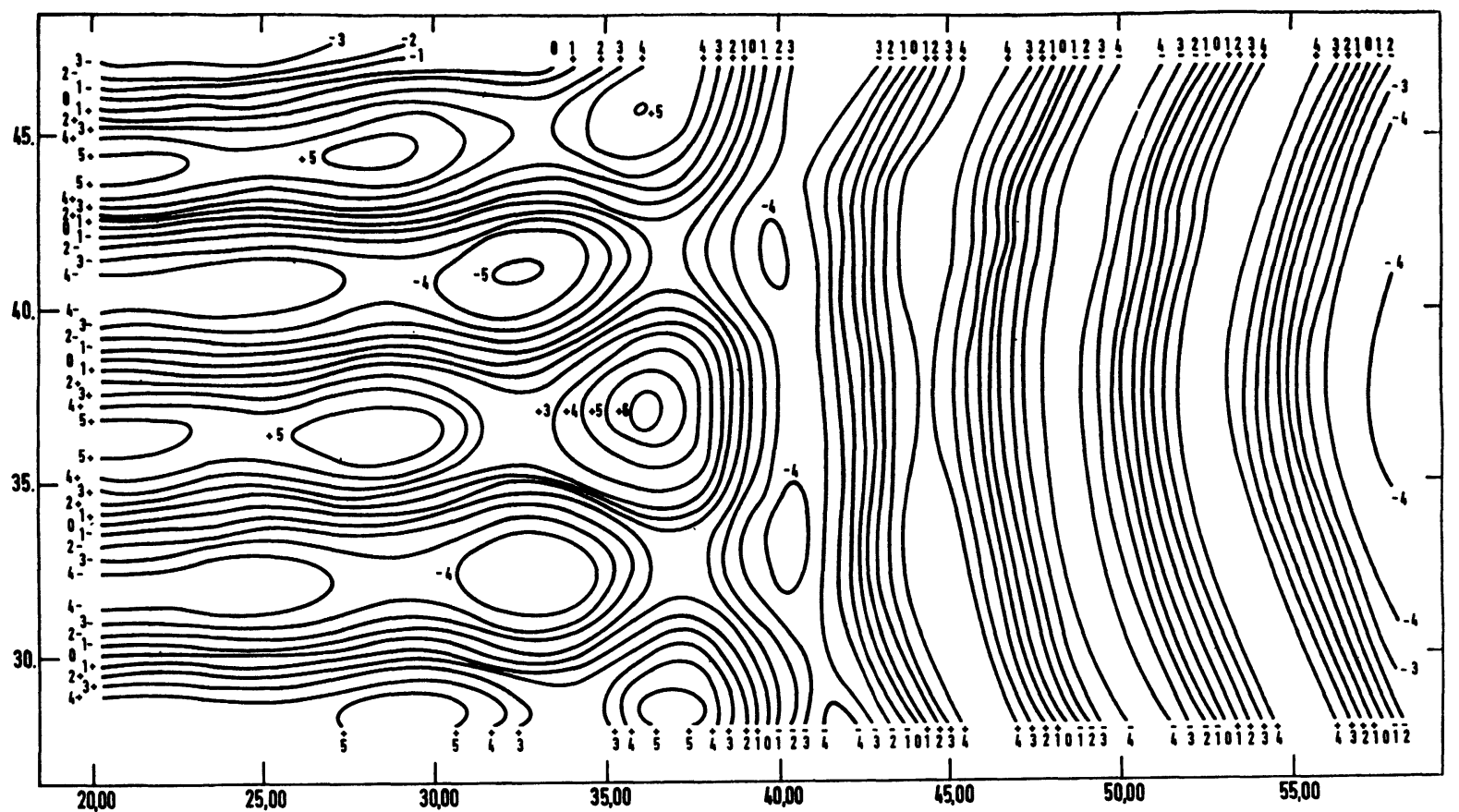

Fig. 9. - Velocity field of a grain boundary. The lines are isovelocity curves. Actually they are associated to the same Doppler shift (multiply the figures by $100 \mathrm{~Hz}, 100 \mathrm{~Hz}=30 \mu \mathrm{m} / \mathrm{s}$ ). This velocity field is build up from 2500 velocity measurements.
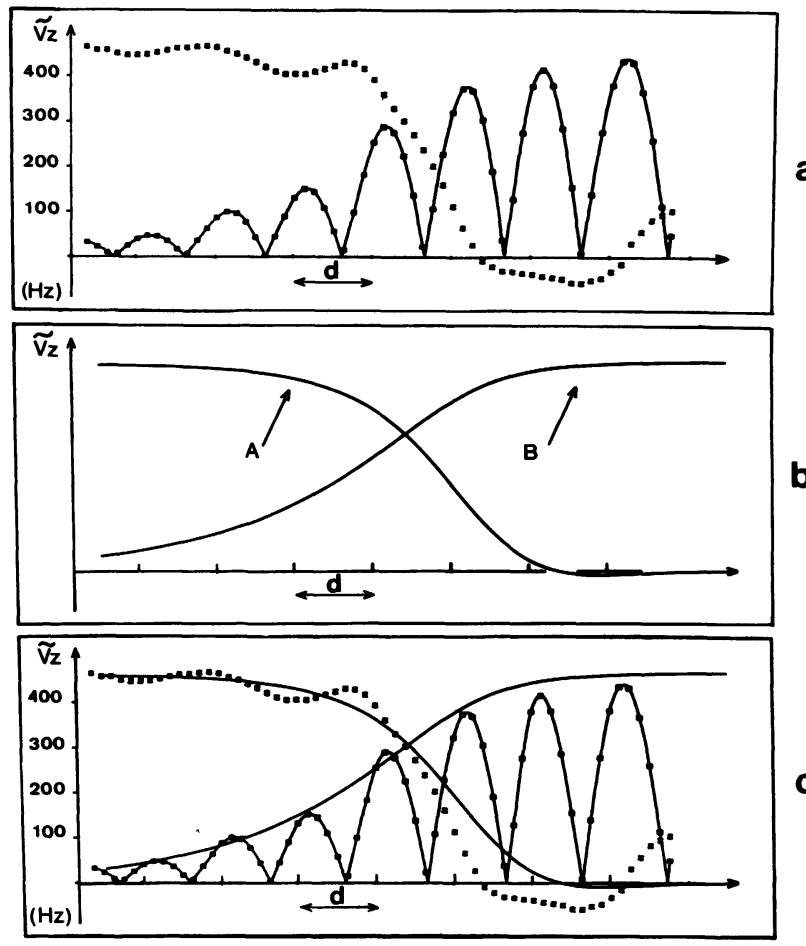

Fig. 10. - Amplitude of the two sets of rolls in the grain boundary. a) Experimental results obtained by averaging the velocity field over one wavelength $(\varepsilon=0.22)$.

b) Computer simulation of the amplitude equation [23].

$$
\begin{aligned}
& \tau_{0} \partial_{\mathrm{t}} A=\varepsilon A-\frac{\xi_{0}^{2}}{2 q_{0}^{2}} \partial_{\times \times \times \times}^{4} A-g A^{3}-\mu A B^{2} \\
& \tau_{0} \partial_{\mathrm{t}} B=\varepsilon B+\xi_{0}^{2} \partial_{\times \times}^{2} B-g B^{3}-\mu A^{2} B
\end{aligned}
$$

made by $\mathbf{P}$. Manneville for the same physical conditions. c) Is $a)+b$ ). to $y=41 \mathrm{~mm}$. The width of this strip corresponds to one wavelength of the A set of rolls, thus by averaging the velocity in the $y$ direction over this length, we cancel the contribution of the A set of rolls so that we a get finally the velocity contribution of the B set only. Plotting the absolute value of this velocity gives us the shape of the amplitude function. By substracting the velocity of the B set of rolls from the total velocity we obtain the velocity field of the A set of rolls. It is easy to deduce its amplitude as a function of $x$, the results appear as given in figure $10 a$. The penetration lengths appear to be clearly different along and perpendicularly to the roll axis : the set of rolls B penetrates deeply the A set of rolls but the contrary is not so noticeable. Y. Pomeau and P. Manneville, using this property have derived an analytic expression of the amplitudes of the two roll structure [26]. The extension of our velocity field is not large enough to allow comparison with the analytic expression. However we reproduce c in figure $10 b$ the amplitude profiles computed on the amplitude equations introduced in [24]; in these simulation the parameters have been set to coincide with our experimental conditions. One parameter, which may be easily obtained, is the penetration length of the B set of rolls in the A set of rolls (the determination of the other opposite penetration length is of poor accuracy). In the following table we reproduce its value for two temperature differences. We add in this table the coherence length $\xi$ calculated for our experimental conditions.

$\begin{array}{ccc}\frac{\varepsilon}{2} & \text { Penetration length } & \text { Coherence length } \\ 0.22 & (1.77 \pm 0.1) d & 0.82 d \\ 0.07 & (4.2 \pm 0.7) d & 1.45 d\end{array}$


We clearly see that the penetration length is about twice the correlation length. This numerical factor is in good agreement with theoretical predictions [26].

9. Stability of the grain boundary. - Starting from the equilibrium structure at $\varepsilon=0.22$ we have reduced

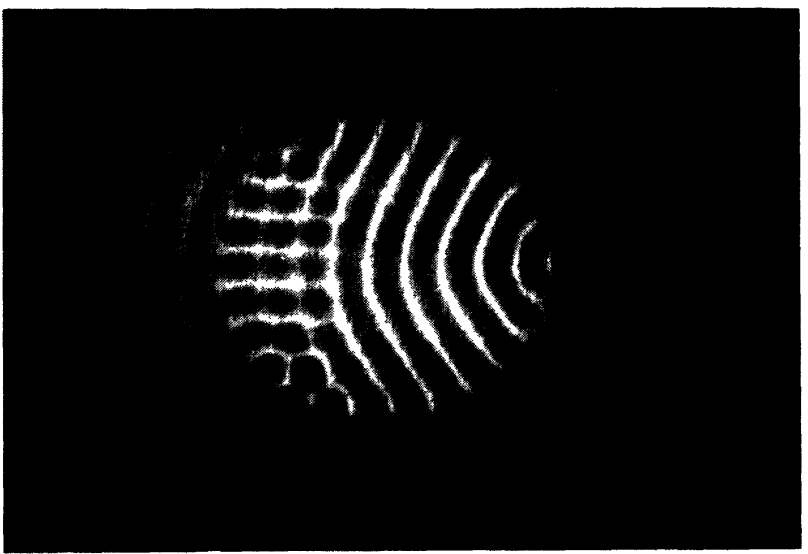

(a)

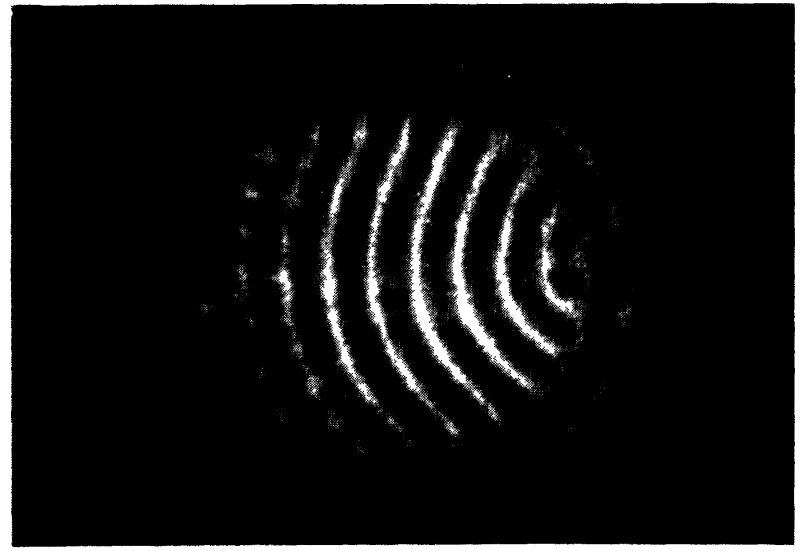

(b)

Fig. 11. - Evolution of the grain boundary when we decrease $\varepsilon$ from 0.22 to 0.07 . The set of rolls B tend to expend at the expense of the A set. $a$ ) Intermediate state. $b$ ) New stationary state. the temperature difference so that $\varepsilon=0.07$. This time we did not record the velocity field but we followed the evolution of the structure by taking pictures (Fig. 11) and by recording the velocity along just one line (Fig. 12). As is expected the penetration lengths increase (see Fig. 12), producing a destabilization of the defect : the A roll structure expands at the expense of the $B$ roll structure (see Fig. 11). This process is rather slow but roll by roll the horizontal structure tends to dominate the overall structure. This appears to be in agreement with the functional point of view described in [15]. At high $\varepsilon$ the number of metastable structures is very important; as $\varepsilon$ decrease this number also decreases leading to some kind of fundamental structure which we believe is made up mainly of one set of rolls like in figure $11 b$. Nevertheless the orientation of these rolls is random and the details of the structure are not universal so that even at low $\varepsilon$ the number of these possible structures is still large.

We may also notice on figure $11 b$ that at some place the rolls do not end perpendicular to the sidewall; and that when they are tilted, another set of rolls appears perpendicularly to the first set [27].

10. Conclusion. - In this article we show that texture-like structures are the usual structures observed in large aspect-ratio containers. These disordered structures appear since they fulfil the two main properties of slightly supercritical structures namely : a relative uniform width of the roll and the general boundary condition for the rolls to end perpendicular to the sidewalls of the container. In particular this last property is responsible for the instability of axisymmetric structures in cylindrical container.

The use of laser Doppler anemometry gives us the possibility of making quantitative comparisons with the amplitude equation : we demonstrate that the amplitude in an axisymmetric container presents a maximum at the centre of the container. We also present an accurate velocity field of a dislocation in a roll structure, and this gives us some insight into the

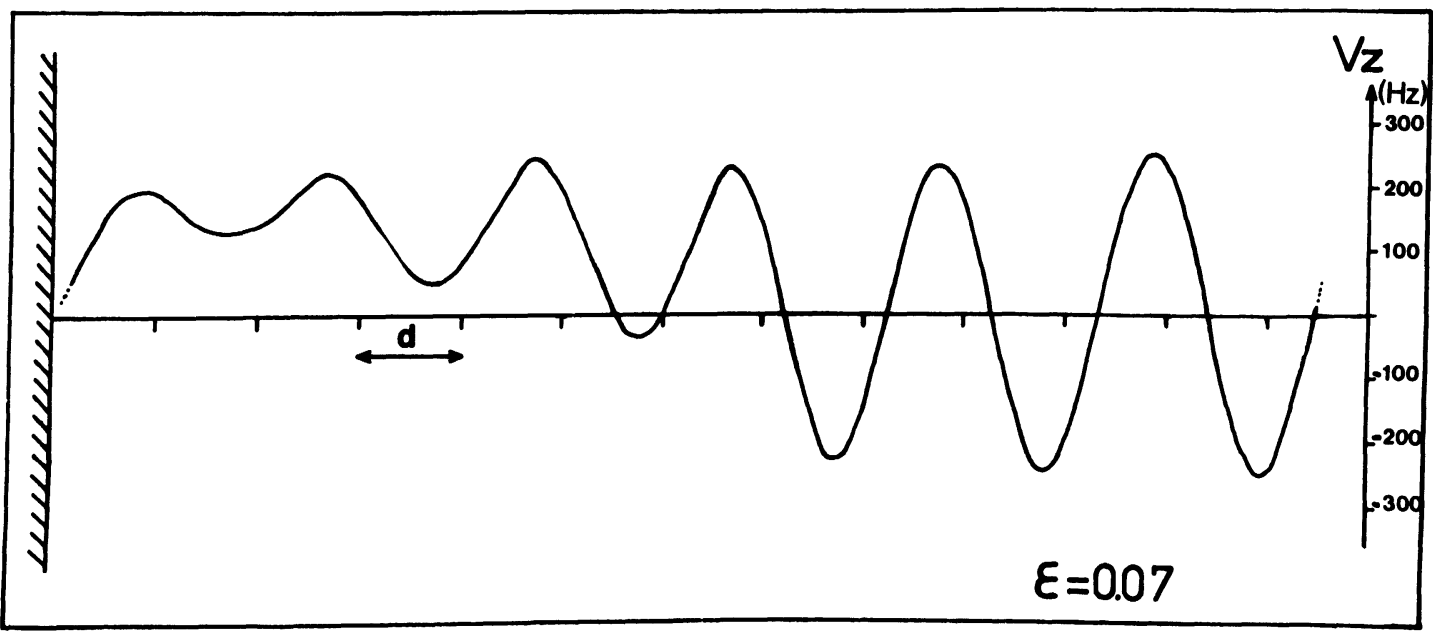

Fig. 12. - Velocity profile of the grain boundary at $\varepsilon=0.07$. The penetration length of the set of roll B becomes very large. 
core region of this defect where the phase field has a singularity. Finally we reproduce the velocity field of a very common defect in Rayleigh-Bénard pattern, namely a grain boundary. This field has to be compared with the amplitude profile of the two sets of rolls calculated by P. Manneville and Y. Pomeau [27]. The stability of this defect is studied as we decrease the Rayleigh number; we show that one set of rolls tends to dominate the overall structure. This set of rolls happens to be the one having the strongest penetration length.
Acknowledgments. - We wish to thank P. Manneville, Y. Pomeau, P. Bergé, M. Dubois, A. Pocheau, J. M. Picquemal, S. Zaleski, J. E. Wesfried and E. Guazelli for fruitful discussions. We have also greatly appreciated the stimulating ideas explained in the series of seminars organized by S. Fauve at the Ecole Normale Supérieure. We are also indebted to C. Poitou, B. Ozenda and M. Labouise for their technical assistance.

\section{References}

[1] Ruelle, D. and Takens, F., Comm. Math. Phys. 20 (1971) 167 ;

Feigenbaum, M. J., Stat. Phys. 19 (1978) 25 ;

Manneville, P. and Pomeau, Y., Physica D 1 (1980) 219; (A review in this field has been given by J. P. Eckman, Rev. Mod. Phys. 53 (1981) 643).

[2] Dubois, M., Bergé, P., J. Physique 42 (1981) 167;

Bergé, P., Dubois, M., Manneville, P., Pomeau, Y., J. Physique Lett. 41 (1980) L-341;

Dubois, M., Bergé, P. and Croquette, V., J. Physique Lett. 43 (1982) L-295;

Maurer, J. and Libchaber, A., J. Physique Lett. 41 (1980) L-515;

Libchaber, A. and Maurer, J., J. Physique Colloq. 41 (1980) C3-51;

Libchaber, A., Laroche, C. and Fauve, S., J. Physique Lett. 43 (1982) L-211;

Giglio, M., Muzzati, S. and Perini, U., Phys. Rev. Lett. 47 (1981) 243;

Gollub, J. P. and Benson, S. V., J. Fluid Mech. 100 (1980) 499.

[3] Ahlers, G. and Beringer, R. P., Phys. Rev. Lett. 40, 11 (1978) 712;

Ahlers, G. and Walden, R. W., Phys. Rev. Lett. 44, L7 (1980) 445.

[4] Libchaber, A. and Maurer, J., J. Physique 39 (1978) 369.

[5] Martinet, B., Haldenwang, P., Labrosse, G., Payan, J. C. et Payan, R., J. Physique Lett. 43 (1982) L-161.

[6] Siggia, E. D. and Zippelius, A., Phys. Rev. Lett. 47 (1981) 835.

[7] Busse, F. H. and WhiteheAd, J. A., J. Fluid Mech. 47 (1971) 305.

[8] Chen, M. M. and Whitehead, J. A., J. Fluid Mech. 31 (1968) 1.

[9] Croquette, V. and Schosseler, F., J. Physique 43 (1982) 51.

[10] Bergé, P., Chaos and Order in Nature (Springer Verlag Berlin-Heidelberg-New York) 1981, p. 14.
[11] Koschmieder, E. L. and Pallas, S. C., J. Heat Mass Trans. 17 (1974) 991.

[12] Hoard, C. Q., Robertson, C. R., Acrivos, A., J. Heat Mass Trans. 13 (1970) 849.

[13] Stork, K. and Muller, U., J. Fluid Mech. 71 (1975) 231.

[14] Kirchartz, K. R., Muller, U., Oertel, H. and ZIEREP, J., Acta Mech. 40 (1981) 181.

[15] Cross, M. C., Phys. Rev. A 25 (1982a) 1665.

[16] Somerscales, E., Dropkin, D., J. Heat Mass Trans. 9 (1966) 1189 ;

Bergé, P., DuboIs, M., Systems for from equilibrium (Springer-Verlag Berlin-Heidelberg-New York) 1980.

[17] Gollub, J. P. and Steinman, J. F., Phys. Rev. Lett. 47 (1981) 505, and preprint submitted to J. Fluid Mech.

[18] Ahlers, G. and Cross, M. C., Hohenberg, P. C. and SAFran, S., J. Fluid Mech. 110 (1981) 297.

[19] Brown, S. N. and Stewartson, F. R. S., Proc. R. Soc. London Ser. A 360 (1978) 455.

[20] Brown, S. N. and Stewartson, F. R. S., SIAM, J. Appl. Math. 36 (1979) 573.

[21] Wesfreid, J., Pomeau, Y., Dubois, M., Normand, C. and Bergé, P., J. Physique 39 (1978) 725 ;

Cross, M. C., Phys. Fluids 23 (1980) 1727.

[22] Siggia, E. D. and Zippelius, A., Phys. Rev. A 24 (1981) 1036.

[23] Pomeau, Y., Zaleski, S., Manneville, P., Phys. Rev. A to appear.

[24] Brown, S. N. and Stewartson, K., Stud. Appl. Math. 57 (1977) 187 ;

Newell, A. C. and Whitehead, J. A., J. Fluid Mech. 38 (1969) 279.

[25] Guazzelli, E., Guyon, E. and Wesfreid, J. E., submitted to Philos. Mg.

[26] Manneville, P., Pomeau, Y., submitted to Philos. Mag.

[27] We are indebted to $S$. Zaleski for having pointed out this effect. 